\title{
TMS Northeast Regional Meeting Considers Processing and Applications of High $\mathrm{T}_{\mathrm{c}}$ Superconductors
}

"Processing and Applications of High $\mathrm{T}_{\mathrm{r}}$ Superconductors: Status and Prospects" was the subject of the May 9-11, 1988 Northeast Regional Meeting of The Metallurgical Society (TMS). The meeting was cosponsored by the Materials Research Society and ASM International. With about 200 attendees and single sessions for two and a half days at Rutgers University (Piscataway, NJ), the meeting had the feeling of a Gordon Conference. It was a pleasure to be able to hear all the papers given and get the full scope of the program. The Conference Organizing Committee was ably chaired by W. E. Mayo (Rutgers University) and included E.A. Giess (IBM and the MRS representative), M.L. Green (AT\&T Bell Laboratories), B. Kear (Rutgers University), J. Lloyd (IBM and the ASM representative), R.S. Polizzotti (Exxon and the TMS New Jersey Chapter chairman), A.S. Rao (University of Cincinnati), and J.B. Watchman (Rutgers University). Support came from the State of New Jersey, Rutgers University Center for Ceramics Research, Exxon Research and Engineering, and IBM Research. Nobel laureate J.R. Schrieffer (University of California, Santa Barbara) started the program with a keynote address covering the development of superconductivity theory.

Each of the five sessions of orally presented papers had at least two invited papers. The first half of the meeting was devoted to all aspects of films, and the latter half to characterization, synthesis, and processing of composites (mainly wires), ceramics, and bulk materials. Posters combined with a vendor exposition nicely filled breaks in the formal sessions.

The conference hotel, near the Rutgers University campus, provided modern rooms and produced a good conference dinner the first night when Dr. A. Schriesheim, director, Argonne National Laboratory, spoke on "Phase Transition from Euphoria to Reality." It was an obviously candid and realistic discussion of the prospects for superconductor technology from an experienced science and engineering manager. A well-established "entry product" is the niobium-titaniumbased superconducting magnet coil in most magnetic resonance imaging units in hospitals around the world. Magnetic levitation, power generators, and special devices (e.g., SQUID amplifiers) pose areas where useful technology could evolve. Mechanical brittleness, environmental corrosion, and critical current limi- tations are problems for the new oxide superconductors, which were almost the only superconductors discussed at this meeting.

\section{Keynote Address}

Prof. Schrieffer's keynote address ideally set the stage with the theoretical foundation of superconductivity before the more applications-oriented papers began. Since the 1911 discovery of Onnes of zero resistance in metals near absolute zero, the rate of discovery and development of theory has not been rapid. Earlier it was speculated that electrons might even become immobile at extremely low temperatures and resistance would become infinite. In 1933, MeissnerOschenfeld found the diamagnetic flux exclusion effect, which is the most definitive evidence of superconductivity. Later, London introduced the notion of flux quantization, and in 1954, Abrikosov proposed vortex lattice theory. Vortices relate to the coherence length and are important to understanding how magnetic field lines penetrate superconductors. Somewhat later, Type II superconductors, which have vortex lattices, were observed experimentally. In practical materials it is now recognized that it is the strength of pinning of these vortices that determines maximum current density. Without pinning, all current would be at the surface, so pinning enables current to exist within the bulk. The cuprate superconductors are type II and have coherence lengths of the order of crystallographic unit cell dimensions. To be effective, pinning sites must, unfortunately, have nearly comparable dimensions.

By 1935, the two-fluid model of superconductivity already existed. It calls for coexisting normal electrons flowing parallel to superconducting electrons that are fewer in number. The BCS theory of Prof. Schrieffer and his colleagues identified electron pairs as being the key to superconductivity (zero resistance). Pairs seem to exist in oxide superconductors, too, but the theoretical question to be answered for these new materials is: What causes the pairs to be bound together?. It is the latter mechanism which separates the different kinds of superconductors. Schrieffer commented that theory has been too "strong" for years. If experiments agreed everyone said, "So what," but if experiments disagreed with theory, they were wrong. J.G. Bednorz and K.A. Müller dared to challenge this with some very in- teresting experiments demonstrating the "possibility" of superconductivity in these new materials. Antiferromagnetism surely plays a role, and both pairing theory and fluctuations are needed to understand the superconductivity of cuprates. Schrieffer further believes a "spin mattress" effect stabilizes pairs where holes have locally destroyed magnetic order. (Picture two people on a soft mattress on a bed.) This fits with the concept that a hole destroys magnetism in a volume called a "spin bag" proposed by Wen and Zhang. More physical understanding is clearly evolving, and this lecture was a highlight of the meeting.

With apologies to the speakers, from this point onward, I am not going to single out any speaker or presentation. Instead, I will attempt to summarize the content and significance of the presentations. A proceedings will be published for the meeting and is scheduled for completion this Fall. Compact and with a good scope, it should be good for someone looking for an overview of the field.

\section{Session Summaries}

The $90 \mathrm{~K}$ material, $\mathrm{Ba}_{2} \mathrm{YCu}_{3} \mathrm{O}_{4}$, also known as "1:2:3" (it should properly be 2:1:3, with divalent Ba listed before trivalent $\mathrm{Y}$ ), understandably received a lot of attention. However, there is more excitement among synthesis and theoretical physics people over the newer higher $T_{\text {c }}$ (106) and $125 \mathrm{~K}$ ) Bi and Tl containing cuprates. Stability relationships are being determined for the different numbers $(1,2$ or 3) of $\mathrm{Cu}-\mathrm{O}$ layers stacked between terminating (capping) $\mathrm{Bi}-\mathrm{O}$ or $\mathrm{Tl}-\mathrm{O}$ layers in the unit cell. These cuprates apparently have only $\mathrm{Cu}-\mathrm{O}$ planes (no chains), showing that chains are not needed for high Tc as was suspected before now. It means more compositions might be useful than if both were required.

The thin films session dealt more with synthesis and characterization than with applications. Films of the new $\mathrm{Bi}$ and $\mathrm{Tl}$ compounds are being grown, but have not been able to match the current densities reported for 1:2:3 epitaxial films. It is evident that a number of workers use fluoride precursors or vacuum techniques to avoid the problem of $\mathrm{BaCO}_{3}$ forming when $\mathrm{Ba}$ in films reacts with atmospheric $\mathrm{CO}_{2}$ or sometimes with reaction products. Films tend to chemically react with atmospheric $\mathrm{CO}_{2}$ or sometimes with reaction products. Films tend to chemically react with substrates and usually are degraded 
especially badly if the dopants replace $\mathrm{Cu}$ ions. Thus, methods allowing synthesis at lower reaction temperatures help avoid this problem and are important.

Because of the planar anisotropy of superconductivity in cuprates, epitaxial films are useful for both theoretical and applications studies. The best synthesis method would seem to be to build films a layer at a time in the desired crystallographic arrangement. Unfortunately, bulk 1:2:3 has to undergo a microcrack generating phase transition in presentday processes. MBE is beginning to be discussed as a film fabrication technique, and is one method for tailoring films that satisfies the above criteria. Films with their short diffusion distances can usually be processed at lower temperatures than bulk materials. While the new $\mathrm{Bi}$ and $\mathrm{Tl}$ compounds do not appear to suffer from unwanted phase changes, they do seem to be more reactive with substrates than the older 1:2:3 compounds.

In forming composites, wires, and ceramics, several difficulties are encountered. The brittleness of superconducting oxides restricts the amount of strain or deformation they tolerate before fracture. Also, structures requiring the coexistence of metals and superconducting oxides are difficult to oxidize enough to achieve superconductivity without altering the metal phase. Most metals have replacement reactions with the copper in the superconducting oxides and seriously degrade superconducting properties.
Even silver reacts with 1:2:3 and can replace copper to a degree, but evidently without serious consequences to superconductivity. Polycrystalline processes where melting occurs make materials with current densities $J_{c}$ two orders higher than sintering processes do. Single crystals give comparable $\mathrm{J}_{\mathrm{c}}$ as the melted materials and do not show as much effect from magnetic field intrusion up to nearly 1 tesla.

Besides the usual difficulties attendant with planar anisotropy in crystals and films, a major problem in all polycrystalline superconductors is electrical connectivity broken grain-to-grain by grain boundary impurities, cracks, or orientation mismatch. The latter effect arises from the electric anisotropy of these materials. Where cost is no object, crystals (and to a lesser degree films) win here. For polycrystals, materials scientists attempt to "texture" structures, i.e., to align neighboring grains in crystallographic orientations favorable to conduction. Mechanical working, e.g. sinter forging, can introduce finer texturing and twinning that enhance pinning and allow higher critical currents. The same can be said for extrusion techniques that also involve mechanical working. In these sessions it seemed that speakers could not tell all...this might be a signal some commercial applications are forthcoming.

Last but not least, superconductors suffer surface resistance effects for high frequency signals, and really can only have
$\mathrm{R}=\mathbf{0}$ throughout the dc applications. Electron pairs also exhibit inertia at high frequencies. In fact, pure oxygen-free copper can have lower surface resistivity than present-day 1:2:3 at liquid nitrogen $\mathrm{T}=77 \mathrm{~K}$ for $\mathrm{GHz}$ signals, although it is expected that improved quality $1: 2: 3$ will ultimately be better. Important studies at high frequency are under way to understand superconductor behavior as a function of magnetic field and of frequency (Surface resistance usually increases with frequency squared.) and of temperature. This area could yield important new applications taking advantage of the unique properties of the new compounds. In general it seems to me that scientists and engineers involved in these applicationsoriented investigations do interact with the materials scientists concerned with synthesis and molecular engineering. It is important for this to be happening.

While no new materials were announced at this meeting, the characterization of the new bismuth and thallium containing compounds was discussed, sometimes actively. Furthermore, it is obvious everyone expects more discoveries in the future. A new era of superconductivity has opened and does not seem to be slowing down after an amazing first two years. However, some real problems must be solved before many new commercial applications are realized.

Edward A. Giess IBM T.J. Watson Research Center

\title{
Scientific Basis for Nuclear Waste Management
}

\author{
October 10-13, 1988 \\ Internationales Congress Centrum \\ Berlin, West Germany
}

\begin{abstract}
Co-sponsored by MRS, the Hahn-Meitner-Institut and other institutions, the 12th International Symposium on the Scientific Basis for Nuclear Waste Management will focus on the science underlying waste forms (vitreous, ceramic, spent fuel, cement), waste isolation (container materials, backfill, repository), and modeling and performance assessment.
\end{abstract}

Contact: Ms. Helga Fuchs, Hahn-Meitner Institut, Berlin GmbH, Glienicker Strasse 100, D1000 Berlin 39, W. Germany; telephone (49) 30 8009-2291; fax (49) 30 8009-2999, or telex 185 763. 